\title{
CONTRIBUIÇÃO Ȧ DATAÇÃO DA FORMAÇÃO ALTER DO CHÃO, BACIA DO AMAZONAS
}

\author{
ROBERTO F. DAEMON*
}

\begin{abstract}
Similarities between the microflora of the Alter do Chão Formation, Amazonas Basin and the Barreirinhas Basin allow the comparison of the taxonomic entities of these basins. Nine palynomorphs, from wells NO-1-AM (Nova Olinda), and AG-1-PA (Alter do Chão), were selected and compared with recent papers of Lima (1971) and Herngreen (1973), from the Barreirinhas Basin.

The conclusion arrived was that the Alter do Chão Formation sedimentation, in the examined area, developed in the Gretaceous, Middle Albian, Cenomanian and Turonian.
\end{abstract}

INTRODUÇÃo Quando de nossos estudos na Bacia do Amazonas, objetivamos, sobretudo, a bioestratigrafia e a paleogeografia do Paleozóico, Siluriano ao Permiano Superior. As seções examinadas da Formação Alter do Chão, sem dúvida, pelo seu conteúdo palinológico, correlacionavam-se aos do Cretáceo e, tentativamente, foram situadas no Cenomaniano/Maestrichtiano.

Desde então, novas informações acumularam-se. A Bacia de Barreirinhas, com polens idênticos aos da Formação Alter do Chão, teve sua bioestratigrafia e geocronologia montadas através dos estudos de Lima (1971).

Não comentamos na época, mas ficava patente que grande parte dos elementos palinológicos da Bacia de Barreirinhas era constituída pela flora continental circunvizinha, carreada para dentro da bacia, fato esse já sugerido por Müller (comunicação verbal).

Recentemente, ao trabalho de Lima (1971), somou-se o de Herngreen (1973), estudando a palinoestratigrafia e taxonomia dos palinomorfos de Barreirinhas, poço de 1-QS-1-MA (Queimadas). É, pois, através da seleção de algumas espécies básicas que enfocamos o problema da idade da Formação Alter do Chão, com características predominantemente continentais.

Coube a Price (1960) detetar pela primeira vez restos fósseis na Formação Alter do Chão, através de dentes de répteis terópodos, acertadamente correlacionados ao Cretáceo.

Reveste-se de interesse a datação dos sedimentos do ciclo intracratônico do Mesozóico brasileiro, principalmente na solução dos problemas paleogeográficos e paleoecológicos.

O primeiro se liga ao problema da tectônica de placas. Quanto melhor a reconstituição de uma determinada área e mais acuradas as datações, mais próximo chegamos do entendimento da problemática da deriva dos continentes.

O segundo, paleoecologia, ajudando na determinação do clima e do ambiente de deposição das camadas, permite delinear-se a paisagem florística e faunística dominantes durante a deposição de uma determinada formação geológica. $\mathrm{E}$ de suma importância para a compreensão global de um ciclo geológico como o Mesozbico, não só no âmbito das correlações inter-regionais, mas também intercontinentais.

Hoje sabemos que a Formação Alter do Chão possui microflora idêntica à do Cretáceo de Barreirinhas, que possui entidades palinológicas similares às da Africa. No âmbito regional, sabemos que essa microflora se estendeu para sul, pelo menos até à região da Bacia de Sergipe-Alagoas. É sem dúvida uma flora que se desenvolveu no continente, como atestam os achados nos poços de NO-1-AM (Nova Olinda) e AG-1-PA (Alter do Chão).

* Lab. de Paleontologia e Palinologia - Univ. Estadual de Maringá 
Desconhecemos a microflora das formações geológicas intracratônicas, do Mesozóico do Brasil meridional e sul. Seu estudo certamente contribuirá em muito para a compreensão dos problemas de correlações bioestratigráficas e datações, clima e paleogeografia num âmbito geológico mais vasto. Caso se confirme a semelhança com os achados do Norte e do Nordeste, estaríamos diante de uma monotonia climática que, em última análise, viria a contribuir na compreensão da geologia. Caso contrário, teríamos limites de clima dentro do Mesozóico que iriam influir não só na geologia, mas também no aspecto florístico e faunístico.

Segundo o recente trabalho de Asmus e Porto (1973), o Atlântico sul teria se formado no Wealdeaniano, portanto antes dos primeiros registros marinhos verificados no Atlântico norte, Aptiano, Bacia de Barreirinhas.

Qual a possível influência paleoclimática e paleogeográfica, acarretada pela formação mais precoce do Atlântico sul? Não temos ainda essa resposta, uma vez que desconhecemos os elementos florísticos ou microflorísticos dessas regiões, fator primordial para o estabelecimento de uma ecologia.

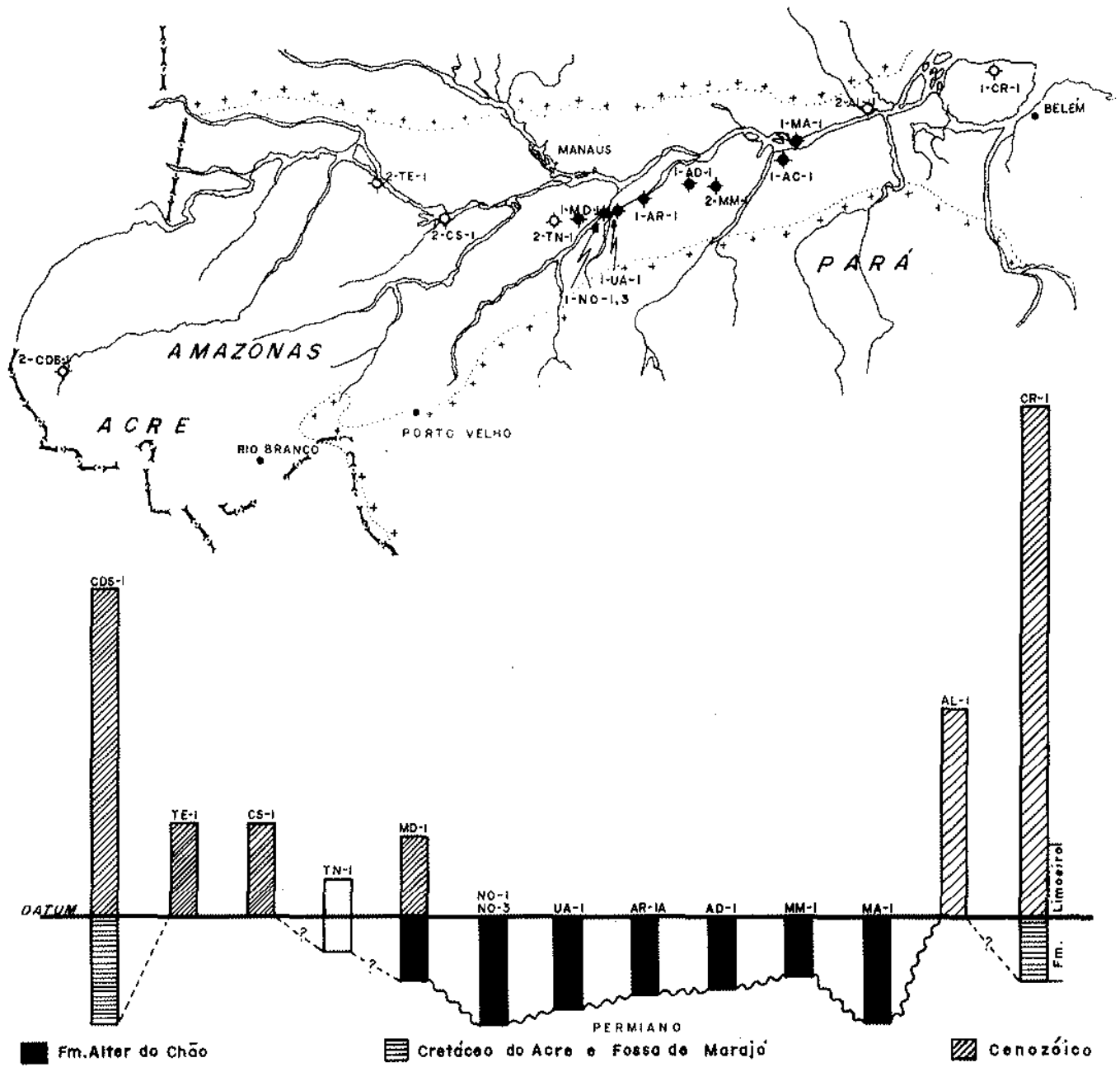

Figura 1 - Mapa de localização dos poços e área de ocorrência da Formação Alter do Chão. Datum, topo do Cretáceo 
Idade da Formação Alter do Chão A seção-tipo da Formação encontra-se no poço I-AC-1-PA (Alter do Chão), nas coordenadas geográficas de $2^{\circ} 31^{\prime} 15^{\prime \prime}$ de latitude sul, e em $54^{\circ} 58^{\prime} 30^{\prime \prime}$ de longitude oeste, segundo Caputo et al. (1971). Nesse poço, a formação alcança $545 \mathrm{~m}$ de espessura.

Segundo os mesmos autores, a litologia é composta por intercalação de arenitos e argilitos e, subordinadamente, conglomerados. Os arenitos geralmente apresentam estratificação cruzada. Os argilitos apresentam-se maciços ou laminados. Os conglomerados constituem paleocanais, com 2 a $5 \mathrm{~m}$ de espessura, que gradam superiormente para arenitos. Nas porções centrais, essa unidade jaz discordantemente sobre a Formação Andirá (Sucunduri), do Permiano Superior.

Segundo constatamos [Daemon e Contreiras (1971)], a formação é encontrada entre os poços 1-Md-1-AM (Madeirinha) e 1-MA-1-PA (Monte Alegre). Em perfil, apresenta-se encravada sobre os sedimentos do Paleozóico e, pelo menos em parte, recoberta pelo Cenozóico (Fig. 1). Suas relações bioestratigráficas com os sedimentos do Cretáceo, dos poços do Acre e da fossa do Marajó, não se encontram bem definidas. Os sedimentos do Mesozóico da fossa de Marajó, Formação Limoeiro com indícios marinhos, parecem ser mais novos que os da Formação Alter do Chão.

Pelos resultados que apresentaremos, afigura-se pouco provável que a deposição da Formação Alter do Chão tenha se processado desde o Cretáceo até o Plioceno, numa deposição ininterrupta.

Cotejando alguns palinomorfos da Formação Alter do Chão (Foto 1), guias do Cretáceo, com os de Lima (1971) e Herngreen (1973), construímos a Tab. I. Os resultados mostram que os testemunhos 23 e 158, respectivamente, dos poços AC-1-MA (Alter do Chão) e NO-1-AM (Nova Olinda), porção basal da formação, indicam como idade provável para o início da deposição o Albiano Médio e o Superior. Segue-se o testemunho 136 do poço NO-1-AM (Nova Olinda), também da parte inferior da formação provavelmente do Cenomaniano Inferior.

O testemunho 83 do mesmo poço, parte média da seção, estaria já situado no Cenomaniano Superior com maiores probabilidades de ser do Turoniano. Assim, na área examinada, a Formação Alter do Chão tem sua sedimentação iniciada no Albiano Médio a Superior, estendendo-se pelo Cenomaniano e Turoniano.

Price (1960) reporta a ocorrência de dentes de réptil terópodo, no intervalo correspondente do testemunho 83 até o testemunho 33 , do poço NO-1-AM, portanto já na parte mais superior da formação. Esse autor menciona a existência de Therapoda na Formação Bauru, nordeste de Cuiabá. Segundo Price (1960), "aqui a Formação Bauru, tida de idade Senoniana, desenvolveu-se largamente na chapada divisora entre as cabeceiras do Rio das Mortes (ou Manso), caudal do Rio Araguaia, e as do Rio Roncador, afluente do Cuiabá, caudal do Rio Paraguai".

Da Bacia do Parnaíba, Formação Itapecuru, Price (1960), de material proveniente da Baía de São Marcos, menciona restos de Theropoda, associados a Sauropoda, Crocodilia, Chelonia, restos de peixes Elasmobranchia, Dipnoi e Actinopterygii, invertebrados, e fragmentos de plantas e madeiras.

Mesner e Wooldridge (1962) consideram a Formação Itapecuru como do Albiano, zonas palinológicas E e F de Müller (1962). Herngreen (1973) compara a sua zona de pólen I à zona $E$ de Müller (1962), situando-a no Albiano Médio.

$\mathrm{Na}$ Tab. II, sumariamos os resultados de idade e paleocologia da Formação Alter do Chão, comparando-os com a Bacia de Barreirinhas, o que, em princípio, não se afigura de todo recomendável, pois trata-se de entidades geológicas distintas. $O$ mesmo não foi 


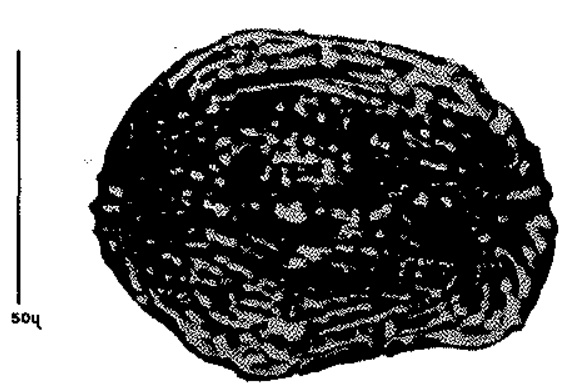

(a)

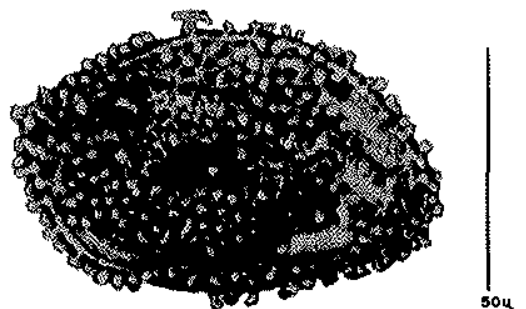

(b)

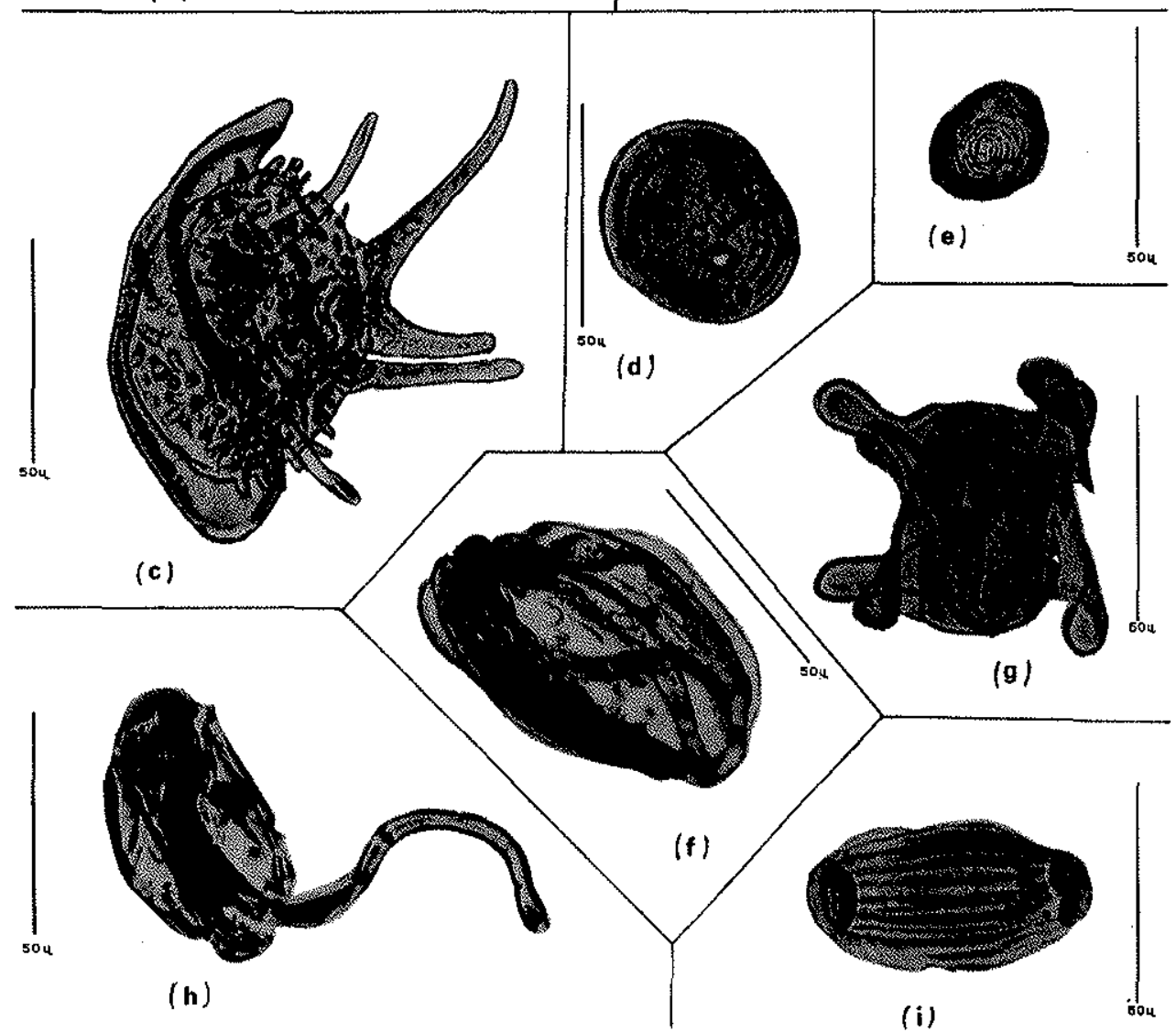

Foto 1 - (a) Gnetaceaepollenites diversus, Stover, 1964. Lâmina 6795/DESUL, Poço NO-1-AM, Test. 83, Fm. Alter do Ghão. (b) Incertae sedis sp. 1, Herngreen, 1973. Formas monocolpadas, esculturadas por clavas. Lâmina 6 796/DESUL, Poço NO-I-AM, Test. 136, Fm. Alter do Chão. (c) Elaterasporites protensus, Stover, 1964. Lâmina 6 783/DESUL, Poço AC-1-Pa, Test. 23, Fm. Alter do Chão. (d) Classopolis classoides, Pflug, 1953. Lâmina 6 797/DESUL, Poço NO-1-AM, Test. 158, Fm. Alter do Chão. (e) Chomotriletes sp. Assemelham-se às formas de Jardiné e Magloire, 1963. Lamina 6791/DESUL, Poço NO-1-AM, Test. 51, Fm. Alter do Chão. (f) Ephedrites irregularis, Herngreen, 1973. Lâmina 6783 /DESUL, Poço AC-1-PA, Test. 23, Fm. Alter do Chão. (g) Elaterocolpites castelaini, Jardiné e Magloire, 1965. Lâmina 6 783/DESUL, Poço NO-1-AM, Test. 83, Fm. Alter do Chão. (h) Elateroplicites africaensis, Herngreen, 1973. Lámina 6 795/DESUL, Poço NO-1-AM, Test. 158, Fm. Alter do Chão. (i) Steevesipollenites binodosus, Stover, 1964. Lâmina 6 796/DESUL, Poço NO-1-AM, Test. 136, Fm. Alter do Chão. 


\begin{tabular}{|c|c|c|c|c|c|c|c|c|c|c|c|c|c|c|c|c|c|c|}
\hline & & & 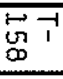 & wi & & & $\bar{\omega}$ & & & & & & & & & & NO-I-MA & D \\
\hline & & $N_{-1}$ & & & & & & & & & & & & & & & $A C-1-P A$ & \\
\hline$\omega r$ & $r$ & $r$ & $r$ & $r$ & $r$ & $r$ & $r$ & 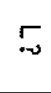 & $\pi$ & $r$ & $r$ & $r$ & \ulcorner & $r$ & $r$ & & $\frac{\text { Classopolis }}{\text { Pflug (1953) }}$ & \\
\hline & & & & & & & $\approx$ & 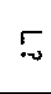 & $r$ & $r$ & $r$ & $r$ & $r$ & \ulcorner & $r$ & & $\frac{\text { Gnetaceaepolienites }}{\text { Stover }(1964)}$ & ersus \\
\hline & $r$ & $\begin{array}{l}r \\
x\end{array}$ & $\begin{array}{l}r \\
x\end{array}$ & $\begin{array}{l}\ulcorner \\
I\end{array}$ & $r$ & $r$ & & & & & & & & & & & $\frac{\text { Steevesipollenites binod }}{\text { Stover }(1964)}$ & dosus \\
\hline & $r$ & $r$ & $\begin{array}{l}r \\
I\end{array}$ & $r$ & $r$ & $r$ & $r$ & $r$ & $r$ & & & & & & & & $\frac{\text { Elaterocolpites }}{\text { Jardiné \& Magloire }(18 \mathrm{e}}$ & \\
\hline & & $\begin{array}{l}r \\
\pm\end{array}$ & $\begin{array}{r}r \\
I\end{array}$ & $\begin{array}{r}r \\
x\end{array}$ &  & 5 & & & & & & & & & & & $\frac{\text { Elaterosporites protens }}{\text { Stover }(1964)}$ & \\
\hline & & & $\begin{array}{l}r \\
\pm\end{array}$ & $r$ & $r$ & r & \ulcorner & $r$ & $r$ & \ulcorner & $r$ & $r$ & $r$ & $r$ & r & & $\frac{\text { Elateroplicites }}{\text { Herngreen }}$ (1973) & \\
\hline & & $I$ & & & & & & & & & & & & & & & $\frac{\text { Ephedrites }}{\text { Herngregularis }}$ & \\
\hline & & $I$ & $I$ & $\sim$ & & & & & & & & & & & & & Chomotriletes sp. & \\
\hline & & $I$ & $I$ & & & & & & & & & & & & & & $\begin{array}{c}\text { Incertae sedis sp. } 1 \\
\text { Herngreen (1973) }\end{array}$ & \\
\hline Aptiano & & lbie & & Cen & man & iano & Tur & oni & & & iac & iano & & toni & iano & Campaniano & $I 0 A D E$ & 要 \\
\hline & $H$ & 3 & $\omega$ & $H$ & 3 & 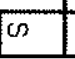 & $H$ & 3 & $\omega$ & $H$ & 3 & To & $H$ & 3 & $\infty$ & \begin{tabular}{|l|l|l|}
$1-1$ & 3 & 0 \\
\end{tabular} & & oै \\
\hline & & & & & & c & R & $E$ & A & c & E & & & & & & PERIODO & 总 \\
\hline & & & & & & E & $s$ & 0 & $z$ & $I$ & c & 0 & & & & & $E R A$ & \\
\hline
\end{tabular}


Tabela II - Correlação bioestratigráfica e paleoecológica entre o Cretáceo das bacias do Amazonas e de Barreirinhas

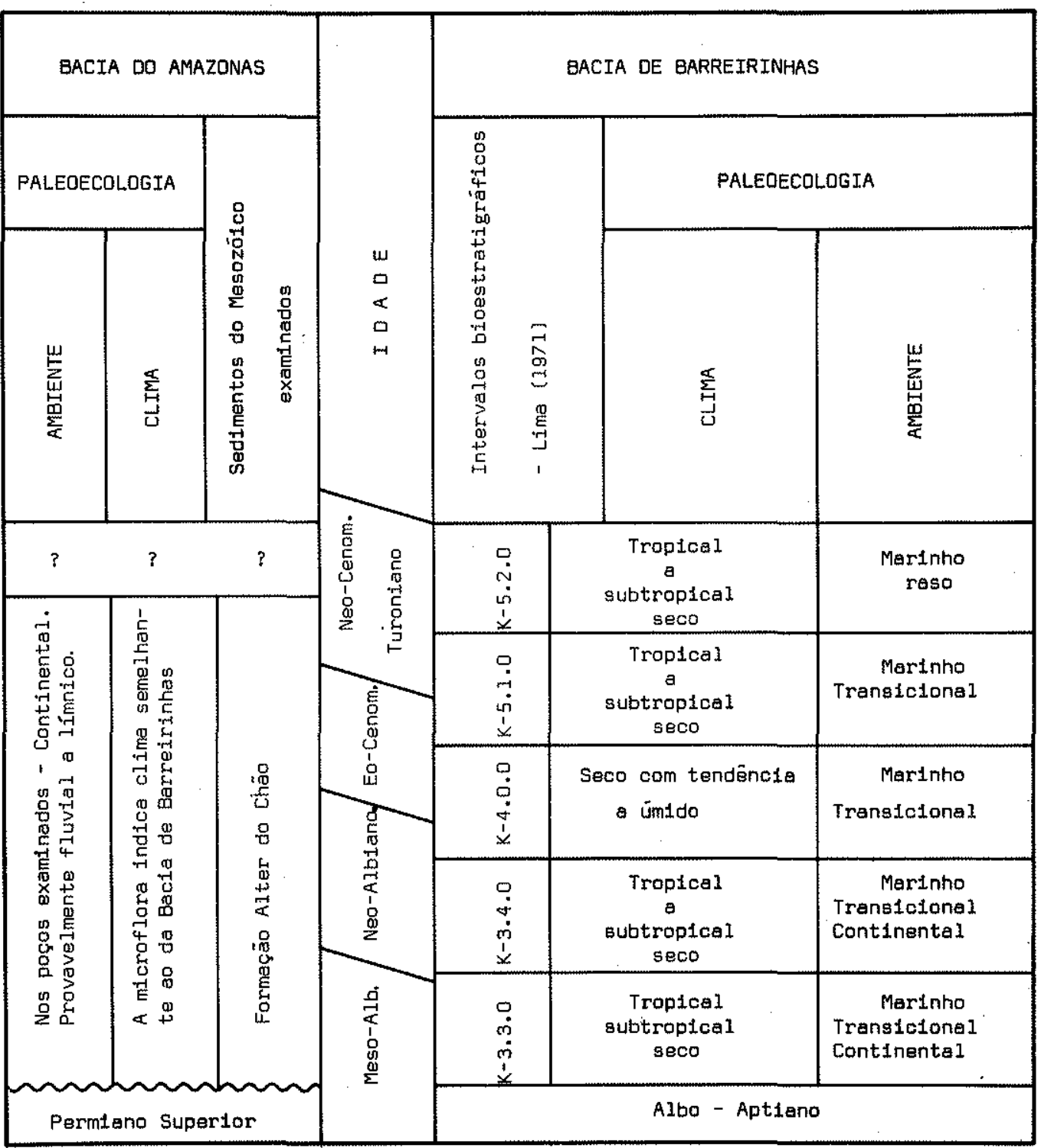

possível fazer em relação ao Mesozóico intracratônico da Bacia do Parnaíba, Bragança - Vizeu e São Luiz, por falta de dados.

Contudo é sugestiva a correlação da Formação Alter do Chão com as formaçōes Itapecuru, Urucuia e Alcântara. Essas formaçðes fazem parte da Sequiência Barro Duro-Arpoador e Bonfim-Preguiças, de Miúra e Barbosa (1972). A primeira, esses autores documentam como amplamente continental; a segunda, com fase marinha mais acentuada com plataformas carbonáticas.

Caso venha a ser comprovada tal hipótese, a Formação Alter do Chão, já não se afigura de interpretação paleogeográfica tão dificil. Em vez de se mostrar como uma entidade 
estratigráfica, confinada ao Amazonas, constituiria uma sedimentação flúvioulacustre, ligada a um sistema hidrográfico de uma pretensa rede de drenagem principal situada mais a sul.

Somente com o falhamento da fossa de Marajó teria havido a captura de grande parte da rede de drenagem para a atual foz do Rio Amazonas, dando inicio ao ciclo sedimentar do Cenozóico. Tal assunto carece de suporte científico, em seu aspecto genético. Desconhecemos mesmo qualquer estudo de paleocorrentes para a Formação Alter do Chão e das suas possíveis correlatas. Contudo a hipótese é válida e deve ser pesquisada. Esse assunto implicaria, a longo prazo, no conhecimento da atual configuração da drenagem do Rio Amazonas e dos problemas ligados ao Cenozóico, tais como clima e vegetação, culminando, em última instância, como uma grande ajuda aos estudos do Quaternário, ainda tão especulativos no Brasil.

Agradecimentos Ao Dr. Setembrino Petri, do Instituto de Geociências da Universidade de São Paulo, pela correção do texto e valiosas sugestôes. À Universidade Estadual de Maringá, pela possibilidade de apresentação deste trabalho no XVIII Congresso Brasileiro de Geologia.

\section{BIBLIOGRAFIA}

AGUIAR, G. A. - 1971 - "Bacia do Maranhão: geologia e possibilidades de petróleo". Petrobrás-RENOR-381

AGUIAR, G. A. - 1971 -- "Revisão geológica da bacia paleozóica do Maranhão". In: Congresso Brasileiro de Geologia, 25, Sða Paulo, 1969, Anais, Sociedade Brasileira de Geologia, v. 3, pp. 113-122

ASMUS, A. E. e PORTO, R. - 1973 - Classificação genética das bacias sedimentares brasileiras segundo a tectônica de placas, Rio de Janeiro, SEPES - Divisão de Desenvolvimento Pessoal, Setor de Documentação, v. 1

CAPUTO, M. V., RODRIGUES, R. e VASCONCELOS, D. N. - 1971 - Nomenclatura estratigráfica da bacia do Amazonas: histórico e atualização. In Congresso Brasileiro de Geologia, 26, Belém, 1970, Anais, Belém, Sociedade Brasileira de Geologia, v. 3, pp. 35 46

DAEMON, R. F. e CONTREIRAS, J. A. - 1971 - Zoneamento palinológico da bacia do Amazonas. In, Congresso Brasileiro de Geologia, 25, São Paulo, 1969. Anais, S. Paulo, Sociedade Brasileira de Geologia, v. 3, pp. 79-88

HERNGREEN, G. F. W. - 1973 - Palynology of albian-cenomanian strata of borehole 1-QS-1. -MA, State of Maranhão, Brazil, Pollen et Spores, Paris, 15 (3-4): 515-555

LIMA, E. G. - 1971 - Bioestratigrafia da bacia de Barreirinhas, Petrobrás-RENOR-381

MENDES, J. C. e PETRI, S. - 1971 - Geologia do Brasil, Rio de Janeiro, INL, MEC

MESMER, J. C. e WOOLDRIDGE, L. G. - 1964 - Maranhð̊o basin and cretaceous coastal basins, North Brazil, Bulletin of American Association of Petroleum Geologists, Tulsa, 48 (9): I 475-1 512

MIÚRA, K. e BARBOSA, J. C. - 1973 - Geologia da plataforma continental do Maranhão, Piaú, Geará e Rio Grande do Norte. Rio de Janeiro, SEPES - Divisão de Desenvolvimento Pessoal, Setor de Documentação, v. 1

MÜLLER, H. - 1962 - Report on palynological results of samples examined from wells in Maranhão, SETEX/RPBa - Relatório-500

PRICE, L. I. - 1960 - Dentes de therapoda num testemunho de sonda no estado do Amazonas, Anais da Academia Brasileira de Ciências, Rio de Janeiro, 32 (1): 79-84

SAMPAIO, A. V. e NORTHFLEET, A. - 1973 - Estratigrafia e correlaçăo das bacias sedimentares brasileiras, Rio de Janeiro, SEPES - Divisão de Desenvolvimento Pessoal - Setor dc Documentação, v. 1 